\title{
Applications of Propargyl Esters of Amino Acids in Solution-Phase Peptide Synthesis
}

\author{
Ramesh Ramapanicker, Rohit Gupta, Rajendran Megha, and Srinivasan Chandrasekaran \\ Department of Organic Chemistry, Indian Institute of Science, Bangalore 560012, India \\ Correspondence should be addressed to Srinivasan Chandrasekaran, scn@orgchem.iisc.ernet.in
}

Received 27 January 2011; Accepted 12 April 2011

Academic Editor: Michal Lebl

Copyright ( $) 2011$ Ramesh Ramapanicker et al. This is an open access article distributed under the Creative Commons Attribution License, which permits unrestricted use, distribution, and reproduction in any medium, provided the original work is properly cited.

\begin{abstract}
Propargyl esters are employed as effective protecting groups for the carboxyl group during solution-phase peptide synthesis. The propargyl ester groups can be introduced onto free amino acids by treating them with propargyl alcohol saturated with $\mathrm{HCl}$. The reaction between propargyl groups and tetrathiomolybdate is exploited to deblock the propargyl esters. The removal of the propargyl group with the neutral reagent tetrathiomolybdate ensures that most of the other protecting groups used in peptide synthesis are untouched. Both acid labile and base labile protecting groups can be removed in the presence of a propargyl ester. Amino acids protected as propargyl esters are employed to synthesize di- to tetrapeptides in solution-phase demonstrating the possible synthetic utilities of the methodology. The methodology described here could be a valuable addition to currently available strategies for peptide synthesis.
\end{abstract}

\section{Introduction}

Several methods are available for the protection of the carboxyl group of amino acids during peptide synthesis [1]. However, with the advent of combinatorial chemistry and with medicinal chemistry developing into a separate branch of science, the use of amino acids having multiple functionalities and which are different from the natural amino acids has become very common. This has also brought up the requirement of additional protecting groups, which are orthogonal to those being used. The $\alpha$-carboxyl groups of amino acids are commonly protected as methyl, benzyl, $t$-butyl, allyl and fluorenylmethyl esters [1]. A useful new protecting group should not only be orthogonal to at least a few of the above esters but also should be complementary to the amino and hydroxy protecting groups used in peptide synthesis.

Various reports from our laboratory have demonstrated the curious reactivity of propargyl systems with tetrathiomolybdate [2]. It has been shown that propargyl ethers [3] and propargyl esters [4] undergo cleavage in the presence of benzyltriethylammonium tetrathiomolybdate (1) to give alcohols and acids respectively. Propargyloxycarbonyl (Poc) group, which can be deprotected with tetrathiomolybdate (1), has been used as an efficient protecting group for amines [5, 6] and alcohols [7-9], and its applications in peptide synthesis have been established $[6,9]$. Herein, we report a systematic study demonstrating the utility of propargyl esters as a protecting group for carboxyl groups in solution-phase peptide synthesis.

\section{Results and Discussion}

Propargyl (Prp) ester derivatives of a number of $N$-protected amino acids were prepared by treating them with propargyl bromide (DMF, $\mathrm{K}_{2} \mathrm{CO}_{3}, 0^{\circ} \mathrm{C}$ ). The propargyl esters were obtained in excellent yields under the conditions employed (Scheme 1, Table 1). As expected these propargyl esters could be deprotected very effectively using 1 equiv of tetrathiomolybdate $\left(1, \mathrm{CH}_{3} \mathrm{CN}, 28^{\circ} \mathrm{C}, 2 \mathrm{~h}\right)$. The reactions were very clean, and the products were obtained in high yields (Scheme 1, Table 1). The side chain carboxyl groups of aspartic acid and glutamic acids could also be protected as propargyl esters and could efficiently be deprotected (entries 9 and 12 in Table 1). It is notable that the propargyl ester of 2-aminoisobutyric acid, which is much hindered compared 
TABle 1: Preparation and deprotection of propargyl esters.

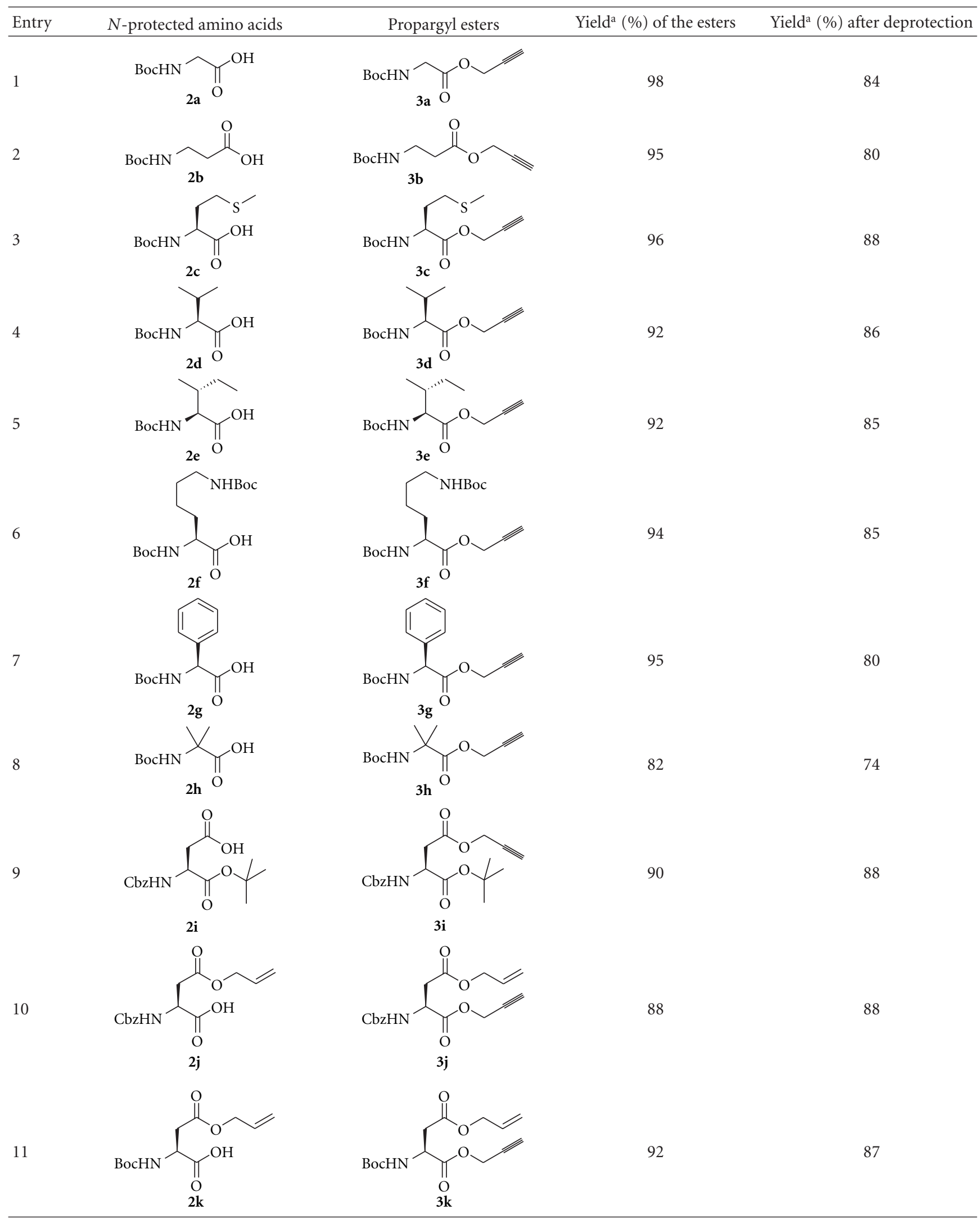


Table 1: Continued.

\begin{tabular}{|c|c|c|c|c|}
\hline Entry & $N$-protected amino acids & Propargyl esters & Yield $^{\mathrm{a}}(\%)$ of the esters & Yield $^{\mathrm{a}}(\%)$ after deprotection \\
\hline 12 & BocHN & $\mathrm{HN}$ & 94 & 83 \\
\hline 13 & $\begin{array}{r}\text { FmocHN } \\
2 \mathbf{m}\end{array}$ & $\mathrm{cHN}$ & 97 & 92 \\
\hline 14 & CbzHN & $\begin{array}{r}\mathrm{O} \\
\mathbf{3 n}\end{array}$ & 81 & 79 \\
\hline 15 & BocHN & $\begin{array}{l}O \\
30\end{array}$ & 94 & 86 \\
\hline
\end{tabular}

${ }^{a}$ The yields reported are of pure compounds isolated through column chromatography.

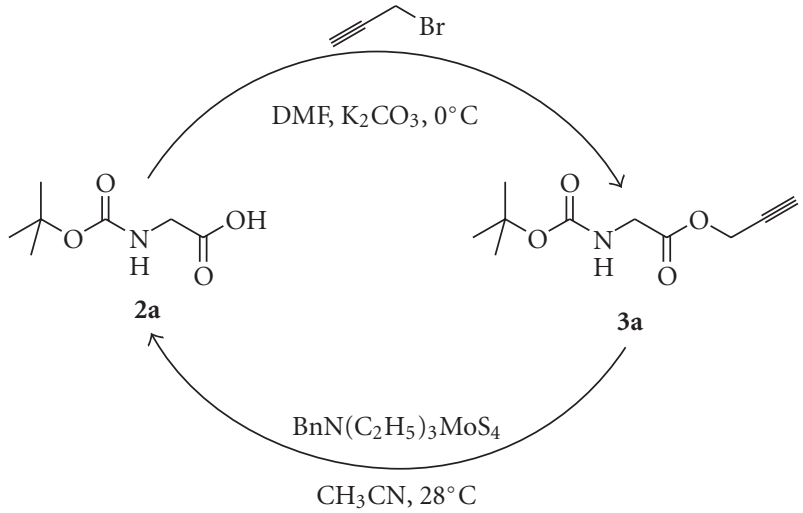

Scheme 1: Preparation of the propargyl ester of Boc-Gly-OH (2a) and the effective deprotection of the propargyl ester with tetrathiomolybdate.

to the other examples studied, could be deprotected in good yield (entry 8, Table 1). Base sensitive Fmoc group in FmocAla-OPrp (3m) was unaffected by the treatment with $\mathbf{1}$, whereas the propargyl ester was deblocked to give $\mathbf{2 m}$ (entry 13, Table 1). The primary amide group of asparagine was also not affected by the conditions used for the deprotection of the propargyl ester (entry 14, Table 1). The deprotection of propargyl ester with 1 leaves a $t$-butyl, an allyl or a methyl ester unaffected (entries 9-12, Table 1). Tetrathiomolybdate (1) is used for the deprotection of propargyl carbonates [79]. However, a benzyl carbonate as in Boc-Tyr $(\mathrm{Cbz})$-OPrp (3o) is unaffected on treatment with $\mathbf{1}$, which removes the propargyl ester selectively (entry 15, Table 1). It has to be noted that Boc-Tyr(Poc)-OPrp (3p), which can be prepared from Boc-Tyr-OH on treatment with PocCl (2 equiv, $\mathrm{Et}_{3} \mathrm{~N}$, $\mathrm{CH}_{2} \mathrm{Cl}_{2},-70^{\circ} \mathrm{C}$ to $\left.\mathrm{rt}, 3 \mathrm{~h}\right)[10]$, reacts with 2 equiv of 1 resulting in the deprotection of both the propargyloxycarbonyl group and the propargyl ester (Scheme 2). The reaction of Boc-Ile-OPrp (3d) with tetrathiomolybdate (1) yielded $\mathbf{2 d}$ in $85 \%$ yield. The reaction did not produce any other diastereomer of Boc-Ile-OH (2d) suggesting that the deprotection of propargyl esters with $\mathbf{1}$ does not result in the racemization of amino acids, as evident from the ${ }^{1} \mathrm{H}$ NMR spectrum of the compound. These results suggest that the propargyl esters of amino acids can be used for the protection of the carboxyl group.

In order for the methodology to be useful in peptide synthesis, it is required that propargyl esters can be prepared from $\mathrm{N}$-unprotected amino acids in good yields. Our efforts to esterify alanine with excess propargyl alcohol in the presence of $\mathrm{SOCl}_{2}$ were unsuccessful. Similarly, reacting alanine and propargyl alcohol in the presence of catalytic amount of $p$-toluenesulfonic acid in benzene, with azeotropic removal of water, was also unsuccessful. The yields of the ester obtained were very poor, and the reaction mixture turned dark, probably from the polymerization of propargyl alcohol. However, when alanine was treated with propargyl alcohol saturated with $\mathrm{HCl}$, propargyl ester of alanine (4a) was obtained in $72 \%$ yield (Scheme 3 ). The procedure was repeated with a number of other amino acids and the corresponding propargyl esters could be isolated in good yields (Table 2 ). The products were initially obtained as brownish 


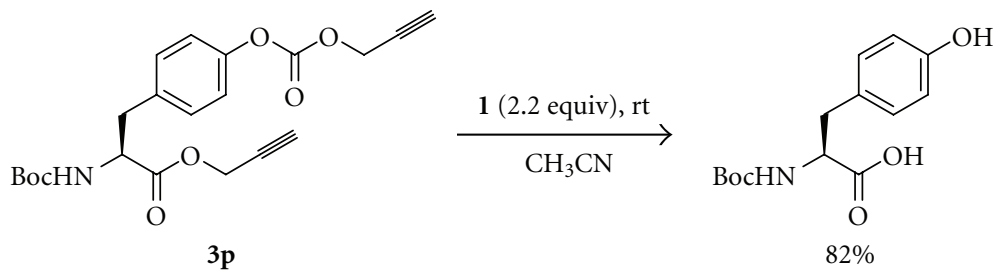

Scheme 2: Simultaneous deprotection of a propargyl ester and a propargyloxycarbonyl group.<smiles>C[C@H](N)C(=O)O</smiles>
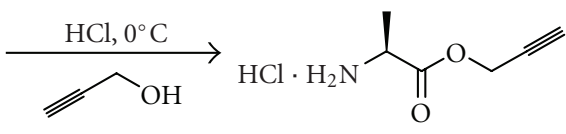

$3 a(72 \%)$

Scheme 3: Preparation of the propargyl ester of alanine.

residues, which had to be washed many times with diethyl ether to remove all the impurities. Valine and isoleucine, which are substituted at the $\beta$-carbon atom, did not react completely. The products were mixtures of the hydrochloride salt of the amino acid and its propargyl ester. The propargyl ester of 2-aminoisobutyric acid (Aib) could not be made even in trace amounts using this procedure. Although substituted at the $\beta$-carbon atom, threonine reacted with propargyl alcohol and $\mathrm{HCl} \cdot \mathrm{H}-\mathrm{Thr}-\mathrm{OPrp}$ (4h) could be isolated in $69 \%$ yield as a single diastereomer, indicating the absence of racemization at the $\alpha$-carbon under these conditions (entry 8 , Table 2), as evident from the ${ }^{1} \mathrm{H}$ NMR spectrum of the compound. The procedure could also be used for preparing the propargyl esters (4i and $\mathbf{4 j}$ ) of 4 - and 3 -aminobenzoic acid in very good yields (entry 9 and 10 , Table 2 ).

The treatment of $\mathrm{HCl} \cdot \mathrm{H}-\mathrm{Ala}-\mathrm{OPrp}(4 \mathrm{a})$ with neat TFA or $20 \%$ piperidine in DMF did not result in the deprotection of the propargyl ester. Therefore, propargyl esters can be used with $t$-butyl based and Fmoc protecting groups. Although propargyl esters can be cleaved using 1 in the presence of an allyl ester (entries 10 and 11, Table 1), cleavage of allyl esters using $\mathrm{Pd}\left(\mathrm{PPh}_{3}\right)_{4}$ and a nucleophile [1] results in the cleavage of propargyl esters (Scheme 4). The results suggest that propargyl esters of amino acids are suitable for solutionphase peptide synthesis, especially when the $\alpha$-amino group is protected as a Boc derivative.

Although propargyl esters of some amino acids could not be prepared directly, they could be prepared from the Boc derivatives of these amino acids (Scheme 1). The cleavage of Boc using TFA in $\mathrm{CH}_{2} \mathrm{Cl}_{2}$ can then provide propargyl esters of such amino acids, which are otherwise difficult to synthesize (Scheme 4). Trifluoroacetic acid salts of the amino propargyl esters thus obtained can directly be used for peptide synthesis. We used this strategy for the preparation of dipeptides from the propargyl esters of Aib, Val, and Ile (Table 3).

Finally to demonstrate the usefulness of the methodology, we synthesized a tetrapeptide through a fragment condensation strategy, which employed the deprotection of propargyl ester with tetrathiomolybdate (1) as one of the key steps. Boc-Ala-OH was coupled with $\mathrm{HCl} \cdot \mathrm{H}-\mathrm{Phe}-\mathrm{OPrp}$ (DCC, $\mathrm{HOBt}, \mathrm{NMM}, \mathrm{CH}_{3} \mathrm{CN}$ ) to get the dipeptide BocAla-Phe-OPrp (6) in $92 \%$ yield. Treating a fraction of $\mathbf{6}$ with TFA ( $50 \%$ in $\mathrm{CH}_{2} \mathrm{Cl}_{2}$ ) gave the free amine TFA. $\mathrm{H}$ Ala-Phe-OPrp (7) and treating another fraction of 6 with tetrathiomolybdate (1) gave the free acid Boc-Ala-Phe-OH (8). The amino component 7 and the carboxyl component 8 were then coupled together (DCC, $\mathrm{HOBt}, \mathrm{NMM}, \mathrm{CH}_{3} \mathrm{CN}$ ) to get the tetrapeptide Boc-Ala-Phe-Ala-Phe-OPrp (9) in $80 \%$ yield (Scheme 5).

\section{Conclusion}

In conclusion, we have demonstrated the utility of propargyl ester as an efficient protecting group for the carboxyl function in solution-phase peptide synthesis. The propargyl ester group is deprotected using the neutral reagent benzyltriethylammonium tetrathiomolybdate, which does not react with other commonly used protecting groups. The introduction or deprotection of the propargyl esters did not result in racemization of the amino acids. Propargyl esters are stable to the conditions used for the deprotection of Fmoc and $t$-butyl-based protecting groups. We have shown the application of the methodology by synthesizing a tetrapeptide.

\section{Experimental}

4.1. General Experimental Procedures. Melting points and optical rotation (at $25^{\circ} \mathrm{C}$ ) were recorded on digital instruments. Infrared spectra were recorded using an FT-IR instrument the frequencies are reported in wave number $\left(\mathrm{cm}^{-1}\right)$, and intensities of the peaks are denoted as s (strong), $\mathrm{w}$ (weak), and $\mathrm{m}$ (medium). ${ }^{1} \mathrm{H}$ and ${ }^{13} \mathrm{C}$ NMR spectra were recorded on a $300 \mathrm{MHz}$ and $75 \mathrm{MHz}$ spectrometer respectively. Chemical shifts are reported in parts per million downfield from the internal reference, tetramethylsilane. Multiplicity is indicated using the following abbreviations: $s$ ( singlet), d (doublet), t (triplet), q (quartet), m (multiplet), dd (double doublet), bs (broad singlet), and bd (broad doublet). Coupling constants are reported wherever it is necessary in Hertz $(\mathrm{Hz})$. Mass spectra were recorded on a High-Resolution Q-TOF electrospray instrument.

4.2. Preparation of Benzyltriethylammonium Tetrathiomolybdate. Ammonium molybdate $(10 \mathrm{~g})$ was dissolved in a mixture of ammonium hydroxide $(60 \mathrm{~mL})$ and water $(20 \mathrm{~mL})$, and the solution was filtered. Hydrogen sulfide was bubbled 


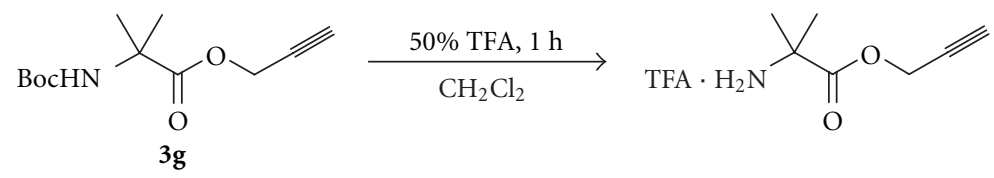

Scheme 4: Preparation of the propargyl ester of Aib from Boc-Aib-OPrp.

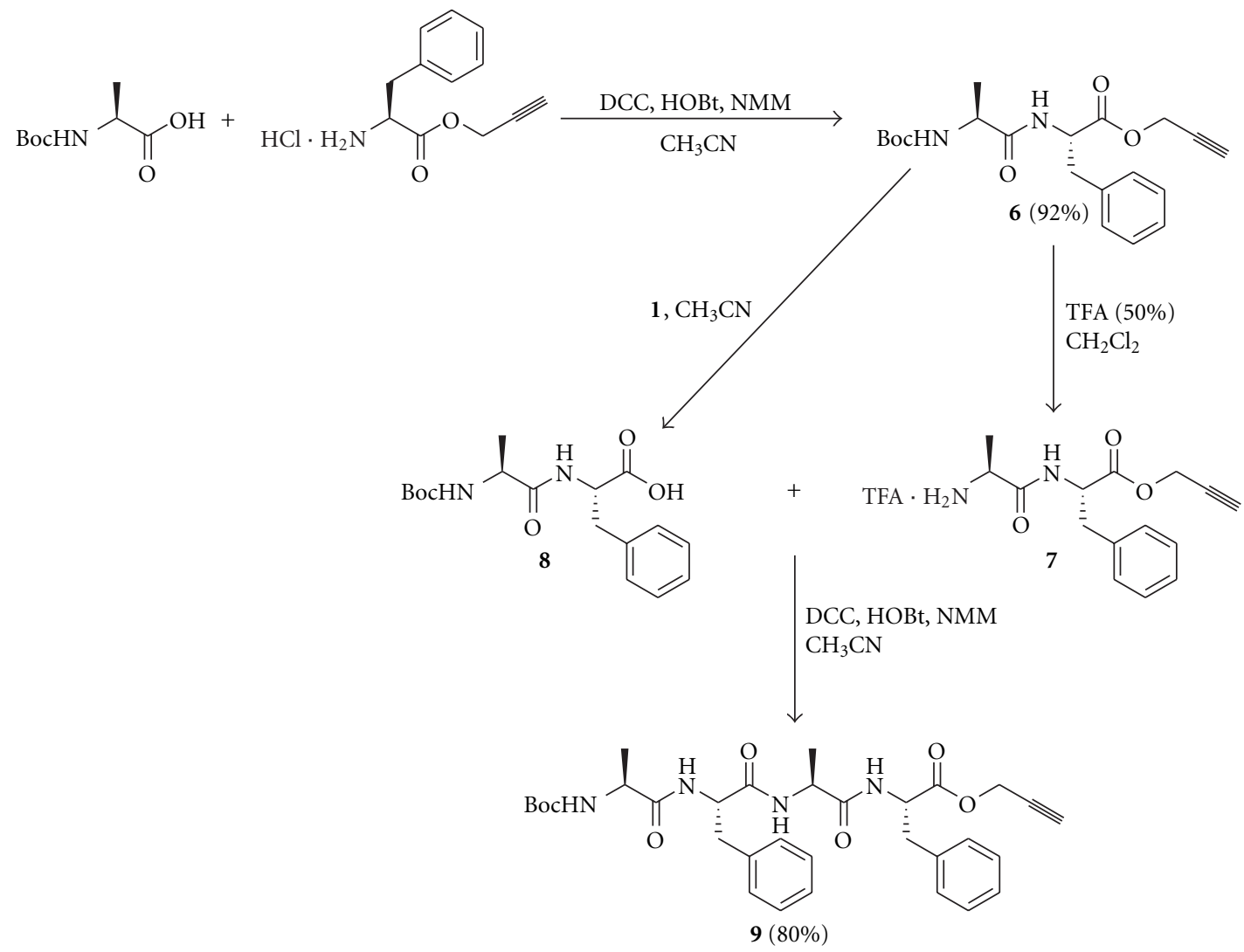

Scheme 5: Synthesis of a tetrapeptide using propargyl ester for carboxyl protection.

rapidly at room temperature $\left(28^{\circ} \mathrm{C}\right)$ into the solution until it was saturated and the temperature was raised to $60^{\circ} \mathrm{C}$, while maintaining a slow stream of hydrogen sulfide. After $60 \mathrm{~min}$, the mixture was cooled to $0^{\circ} \mathrm{C}$ and kept under refrigeration for $30 \mathrm{~min}$. The granular product thus obtained was isolated by filtration. The crystalline solid was washed with isopropyl alcohol $(25 \mathrm{~mL} \times 2)$, ether $(25 \mathrm{~mL} \times 4)$, and dried under vacuum to get brick red crystals of ammonium tetrathiomolybdate (13.4 g, 92\%).

A solution of benzyltriethylammonium chloride (23. $31 \mathrm{~g}, 102.5 \mathrm{mmol})$ in distilled water $(60 \mathrm{~mL})$ was added in portions over $30 \mathrm{~min}$ to a well-stirred solution of ammonium tetrathiomolybdate $(13 \mathrm{~g}, 50 \mathrm{~mL})$ in distilled water $(60 \mathrm{~mL})$. Rapid stirring was continued for $2 \mathrm{~h}$ at room temperature, and the solid that separated was filtered, washed with isopropyl alcohol $(40 \mathrm{~mL} \times 2)$ and ether $(40 \mathrm{~mL}$ $\times 4)$. The brick red powder of benzyltriethylammonium tetrathiomolybdate (1) was dried under vacuum and stored in a desiccator $(24 \mathrm{~g}, 80 \%)$. Melting point: decomposes at $150^{\circ} \mathrm{C}$.

4.3. General Procedure for the Synthesis of Propargyl Esters (3a-o) of N-Protected Amino Acids. N-protected amino acids (2a-o, $5 \mathrm{mmol})$ were dissolved in anhydrous DMF $(10 \mathrm{~mL})$ and the solution was cooled to $-10^{\circ} \mathrm{C}$. Anhydrous $\mathrm{K}_{2} \mathrm{CO}_{3}$ ( $5 \mathrm{mmol}$ ) was added to the solution and the stirring was continued until a syrupy solution is formed. Propargyl bromide $(0.55 \mathrm{~mL}$ of $80 \%$ solution in toluene, $5 \mathrm{mmol}$ ) was added dropwise to the reaction mixture, and the stirring continued at $-10^{\circ} \mathrm{C}$ for $1 \mathrm{~h}$. The reaction mixture was then allowed to attain rt, DMF was removed under vacuum, and the residue was extracted with ethyl acetate $(50 \mathrm{~mL})$. The solution of the crude product was taken in a separating funnel and washed with saturated citric acid solution $(2 \times$ $25 \mathrm{~mL})$, water $(2 \times 25 \mathrm{~mL})$, and brine $(25 \mathrm{~mL})$, dried over anhydrous $\mathrm{Na}_{2} \mathrm{SO}_{4}$ and concentrated. The crude products 
TABLE 2: Preparation of propargyl esters of amino acids.

\begin{tabular}{|c|c|c|c|}
\hline Entry & Amino acid & Amino propargyl ester & Yield $^{\mathrm{a}}(\%)$ \\
\hline 1 & H-Ala-OH & $\mathrm{HCl} \cdot \mathrm{H}$ & 72 \\
\hline 2 & H-Gly-OH & & 69 \\
\hline 3 & H-leu-OH & & 76 \\
\hline 4 & H-Pro-OH & $\mathrm{HCl} \cdot \mathrm{H}$ & 71 \\
\hline 5 & $\mathrm{H}-\mathrm{Ser}-\mathrm{OH}$ & 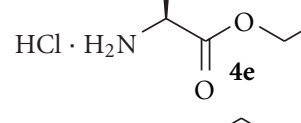 & 70 \\
\hline 6 & H-Phe-OH & 0 & 76 \\
\hline 7 & H-Glu-OH & & 62 \\
\hline 8 & H-Thr-OH & & 69 \\
\hline 9 & 4-ABA & III & 88 \\
\hline 10 & 3-ABA & & 90 \\
\hline
\end{tabular}

${ }^{a}$ The yields reported are of pure compounds isolated after multiple washings with diethyl ether.

were then purified using column chromatography (silica gel, $100-200$ mesh) using a solution of ethyl acetate (10-30\%) in petroleum ether as eluent.

Boc-Gly-OPrp (3a). White crystalline solid; mp $80^{\circ} \mathrm{C}$, FTIR (Neat) 3355 (br), 2131 (w), 1757 (s), 1715 (s); ${ }^{1} \mathrm{H}$ NMR $\left(\mathrm{CDCl}_{3}\right) \delta 5.06(\mathrm{bs}, 1 \mathrm{H}), 4.75(\mathrm{~d}, J=2.4 \mathrm{~Hz}, 2 \mathrm{H}), 3.96(\mathrm{~d}, J=$
$6 \mathrm{~Hz}, 2 \mathrm{H}), 2.51(\mathrm{t}, J=2.4 \mathrm{~Hz}, 1 \mathrm{H}), 1.45(\mathrm{~s}, 9 \mathrm{H}) ;{ }^{13} \mathrm{C} \mathrm{NMR}$ $\left(\mathrm{CDCl}_{3}\right) \delta 169.7,155.6,100.5,80.1,75.4,52.6,42.3,28.3$; ESMS Calculated for $\mathrm{C}_{10} \mathrm{H}_{15} \mathrm{NO}_{4}+\mathrm{Na}$ : 236.2202, Observed 236.2199 .

Boc- $\beta$ Ala-OPrp (3b). Pale yellow oil; FTIR (Neat) 3370 (br), 2129 (w), 1742 (s), 1708 (s); ${ }^{1} \mathrm{H}$ NMR $\left(\mathrm{CDCl}_{3}\right) \delta 5.20$ (bs, 
Table 3: Preparation of dipeptides from propargyl esters of Aib, Val and Ile.

\begin{tabular}{|c|c|c|c|}
\hline Entry & $\begin{array}{l}N \text {-Boc amino propargyl } \\
\text { ester used }\end{array}$ & Dipeptide & Yield $^{\mathrm{a}}(\%)$ \\
\hline 1 & $2 \mathrm{~g}$ & $5 a$ & 67 \\
\hline 2 & $2 c$ & $5 b$ & 82 \\
\hline 3 & $2 \mathrm{~d}$ & $5 c$ & 88 \\
\hline
\end{tabular}

${ }^{a}$ The yields reported are of pure compounds isolated through column chromatography.

$1 \mathrm{H}), 4.70(\mathrm{~d}, J=2.4 \mathrm{~Hz}, 2 \mathrm{H}), 3.40(\mathrm{q}, J=6.3 \mathrm{~Hz}, 2 \mathrm{H}), 2.58$ $(\mathrm{d}, J=6.3 \mathrm{~Hz}, 2 \mathrm{H}), 2.54(\mathrm{t}, J=2.4 \mathrm{~Hz} .1 \mathrm{H}), 1.43(\mathrm{~s}, 9 \mathrm{H})$; ${ }^{13} \mathrm{C} \mathrm{NMR}\left(\mathrm{CDCl}_{3}\right) \delta 171.4,155.5,79.1,74.9,51.8,35.8$, 34.2, 28.1; ESMS Calculated for $\mathrm{C}_{11} \mathrm{H}_{17} \mathrm{NO}_{4}+\mathrm{Na}$ : 250.2468, Observed 250.2465.

Boc-Met-OPrp (3c). Pale yellow oil; $[\alpha]_{\mathrm{D}}-35$ (c=1, EtOH); FTIR (Neat) 3357 (br), 2129 (w), 1749 (s), 1714 (s); ${ }^{1} \mathrm{H}$ NMR $\left(\mathrm{CDCl}_{3}\right) \delta 5.19(\mathrm{bd}, J=7.2 \mathrm{~Hz}, 1 \mathrm{H}), 4.79\left(\mathrm{dd}, J_{1}=15 \mathrm{~Hz}, J_{2}\right.$ $=2.4 \mathrm{~Hz}, 1 \mathrm{H}), 4.70\left(\mathrm{dd}, J_{1}=15 \mathrm{~Hz}, J_{2}=2.4 \mathrm{~Hz}, 1 \mathrm{H}\right), 4.44-$ $4.46(\mathrm{~m}, 1 \mathrm{H}), 2.56(\mathrm{t}, J=7.8 \mathrm{~Hz}, 1 \mathrm{H}), 2.09-2.19(\mathrm{~m}, 4 \mathrm{H})$, 1.91-2.03 (m, 1H), $1.45(\mathrm{~s}, 9 \mathrm{H}) ;{ }^{13} \mathrm{C} \mathrm{NMR}\left(\mathrm{CDCl}_{3}\right) \delta 171.5$, 155.2, 80.1, 75.40, 75.37, 52.7, 31.8, 29.8, 28.2, 15.40, 15.36; ESMS Calculated for $\mathrm{C}_{13} \mathrm{H}_{21} \mathrm{NO}_{4} \mathrm{~S}+\mathrm{Na}$ : 310.3649, Observed 310.3649 .

Boc-Val-OPrp (3d). Pale yellow oil; $[\alpha]_{\mathrm{D}}-36(\mathrm{c}=1, \mathrm{EtOH})$; FTIR (Neat) 3388 (br), 2129 (w), 1747 (s), 1714 (s); ${ }^{1} \mathrm{H}$ NMR $\left(\mathrm{CDCl}_{3}\right) \delta 5.02(\mathrm{bs}, 1 \mathrm{H}), 4.79\left(\mathrm{dd}, J_{1}=15.4 \mathrm{~Hz}, J_{2}=1.8 \mathrm{~Hz}\right.$, $1 \mathrm{H}), 4.68\left(\mathrm{dd}, J_{1}=15.3 \mathrm{~Hz}, J_{2}=1.8 \mathrm{~Hz}, 1 \mathrm{H}\right), 4.24-4.29(\mathrm{~m}$, $1 \mathrm{H}), 2.47-2.50(\mathrm{~m}, 1 \mathrm{H}), 2.12-2.23(\mathrm{~m}, 1 \mathrm{H}), 1.45(\mathrm{~s}, 9 \mathrm{H})$, $0.98(\mathrm{~d}, J=6.9 \mathrm{~Hz}, 3 \mathrm{H}), 0.92(\mathrm{~d}, J=7.2 \mathrm{~Hz}, 3 \mathrm{H}) ;{ }^{13} \mathrm{C} \mathrm{NMR}$ $\left(\mathrm{CDCl}_{3}\right) \delta 171.6,155.6,79.9,77.2,75.2,58.4,52.3,31.3,28.3$, 18.9, 17.5; ESMS Calculated for $\mathrm{C}_{13} \mathrm{H}_{21} \mathrm{NO}_{4}+\mathrm{Na}$ : 278.2999, Observed 278.3001 .

Boc-Ile-OPrp (3e). Pale yellow oil; $[\alpha]_{\mathrm{D}}-31$ (c=1, EtOH); FTIR (Neat) 3385 (br), 2130 (w), 1748 (s), 1714 (s); ${ }^{1}$ H NMR $\left(\mathrm{CDCl}_{3}\right) \delta 5.04(\mathrm{bd}, J=7.8 \mathrm{~Hz}, 1 \mathrm{H}), 4.80\left(\mathrm{dd}, J_{1}=15.4 \mathrm{~Hz}\right.$, $\left.J_{2}=2.1 \mathrm{~Hz}, 1 \mathrm{H}\right), 4.67\left(\mathrm{dd}, J_{1}=15.4 \mathrm{~Hz}, J_{2}=2.1 \mathrm{~Hz}, 1 \mathrm{H}\right)$, 4.28-4.33 (m, 1H), 2.49 (t, $J=2.1 \mathrm{~Hz}, 1 \mathrm{H}), 1.89$ (bs, $1 \mathrm{H})$, 1.39-1.49 (m, 10H), 1.11-1.27 (m, 1H), 0.90-0.96 (m, 6H); ${ }^{13} \mathrm{C} \mathrm{NMR}\left(\mathrm{CDCl}_{3}\right) \delta 171.6,155.5,79.9,77.2,75.1,57.8,52.3$,
38.1, 28.3 , 25.0, 15.5, 11.5; ESMS Calculated for $\mathrm{C}_{14} \mathrm{H}_{23} \mathrm{NO}_{4}$ + Na: 292.3265, Observed 292.3269.

$B o c-L y s(B o c)-O P r p(3 f)$. Pale yellow oil; $[\alpha]_{\mathrm{D}}-24(\mathrm{c}=1$, EtOH); FTIR (Neat) 3353 (br), 2128 (w), 1748 (s), 1697 (s); ${ }^{1} \mathrm{H}$ NMR $\left(\mathrm{CDCl}_{3}\right) \delta 5.17(\mathrm{bd}, J=6.6 \mathrm{~Hz}, 1 \mathrm{H}), 4.79(\mathrm{dd}$, $\left.J_{1}=15.4 \mathrm{~Hz}, J_{2}=2.1 \mathrm{~Hz}, 1 \mathrm{H}\right), 4.68\left(\mathrm{dd}, J_{1}=15.4 \mathrm{~Hz}, J_{2}=\right.$ $2.1 \mathrm{~Hz}, 1 \mathrm{H}), 4.28-4.35(\mathrm{~m}, 1 \mathrm{H}), 3.08-3.14(\mathrm{~m}, 2 \mathrm{H}), 2.52(\mathrm{t}$, $J=2.4 \mathrm{~Hz}, 1 \mathrm{H}), 1.63-1.89(\mathrm{~m}, 2 \mathrm{H}), 1.35-1.60(\mathrm{~m}, 22 \mathrm{H}) ;{ }^{13} \mathrm{C}$ NMR $\left(\mathrm{CDCl}_{3}\right) \delta 172.0,156.0,155.4,79.9,79.1,77.1,75.3$, 53.1, 52.5, 39.9, 32.0, 29.5, 28.3, 28.2, 22.3; ESMS Calculated for $\mathrm{C}_{19} \mathrm{H}_{32} \mathrm{~N}_{2} \mathrm{O}_{6}+\mathrm{Na}$ : 407.4570, Observed 407.4567.

Boc-Phg-OPrp (3g). White solid; $\operatorname{mp} 48^{\circ} \mathrm{C} ;[\alpha]_{\mathrm{D}}+32(\mathrm{c}=1$, EtOH); FTIR (Neat) 3402 (br), 2131 (w), 1748 (s), 1722 (s); ${ }^{1} \mathrm{H}$ NMR $\left(\mathrm{CDCl}_{3}\right) \delta 7.32-7.38(\mathrm{~m}, 5 \mathrm{H}), 5.51(\mathrm{bd}, J=7 \mathrm{~Hz}$, $1 \mathrm{H}), 5.36(\mathrm{bd}, J=7.2 \mathrm{~Hz}, 1 \mathrm{H}), 4.75\left(\mathrm{dd}, J_{1}=16 \mathrm{~Hz}, J_{2}=\right.$ $1.8 \mathrm{~Hz}, 1 \mathrm{H}), 4.67\left(\mathrm{dd}, J_{1}=15 \mathrm{~Hz}, J_{2}=1.8 \mathrm{~Hz}, 1 \mathrm{H}\right), 2.45(\mathrm{t}, J=$ $2.1 \mathrm{~Hz}, 1 \mathrm{H}), 1.43(\mathrm{~s}, 9 \mathrm{H}) ;{ }^{13} \mathrm{C} \mathrm{NMR}\left(\mathrm{CDCl}_{3}\right) \delta 170.4,154.7$, 136.3, 128.9, 128.6, 127.2, 80.3, 76.8, 75.4, 57.5, 53.0, 28.2; ESMS Calculated for $\mathrm{C}_{16} \mathrm{H}_{19} \mathrm{NO}_{4}+\mathrm{Na}$ : 312.3162, Observed 312.3158 .

Boc-Aib-OPrp (3h). Pale yellow oil; FTIR (Neat) 3377 (br), 2130 (w), 1746 (s), 1713 (s); ${ }^{1} \mathrm{H}$ NMR $\left(\mathrm{CDCl}_{3}\right) \delta 5.09$ (bs, $1 \mathrm{H}), 4.72(\mathrm{~d}, J=2.7 \mathrm{~Hz}, 2 \mathrm{H}), 2.47(\mathrm{t}, J=2.7 \mathrm{~Hz}, 1 \mathrm{H}), 1.51$ $(\mathrm{s}, 6 \mathrm{H}), 1.44(\mathrm{~s}, 6 \mathrm{H}) ;{ }^{13} \mathrm{C} \mathrm{NMR}\left(\mathrm{CDCl}_{3}\right) \delta 173.9,154.5,79.8$, 75.0, 56.0, 52.7, 28.2, 25.3; ESMS Calculated for $\mathrm{C}_{12} \mathrm{H}_{19} \mathrm{NO}_{4}$ + Na: 264.2734, Observed 264.2735.

Cbz-Asp(OPrp)-Ot Bu (3i). Colorless oil; $[\alpha]_{D}+83$ (c = 1, EtOH); FTIR (Neat) 3293 (br), 2129 (w), 1738 (s), 1732 (s), $1728(\mathrm{~s}) ;{ }^{1} \mathrm{H} \mathrm{NMR}\left(\mathrm{CDCl}_{3}\right) \delta 7.32-7.36(\mathrm{~m}, 5 \mathrm{H}), 5.76(\mathrm{bd}$, $J=7.5 \mathrm{~Hz}, 1 \mathrm{H}), 5.11$ (s, 2H), 4.50-4.67 (m, 3H), 3.01(dd, 
$\left.J_{1}=17 \mathrm{~Hz}, J_{2}=4.5 \mathrm{~Hz}, 1 \mathrm{H}\right), 2.86\left(\mathrm{dd}, J_{1}=17 \mathrm{~Hz}, J_{2}=4.5 \mathrm{~Hz}\right.$, $1 \mathrm{H}), 1.45(\mathrm{~s}, 9 \mathrm{H}) ;{ }^{13} \mathrm{C} \mathrm{NMR}\left(\mathrm{CDCl}_{3}\right) \delta 169.9,169.2,155.8$, 136.1, 128.4, 128.04, 127.96, 82.6, 75.2, 66.9, 52.2, 50.7, 36.6, 27.7; ESMS Calculated for $\mathrm{C}_{19} \mathrm{H}_{23} \mathrm{NO}_{6}+\mathrm{Na}$ : 384.1423, Observed 384.1426.

Cbz-Asp(OAllyl)-OPrp (3j). White solid; mp $50^{\circ} \mathrm{C} ;[\alpha]_{\mathrm{D}}$ -11 (c = 1, EtOH); FTIR (Neat) 3365 (br), 2130 (w), 1735 (s); ${ }^{1} \mathrm{H} \mathrm{NMR}\left(\mathrm{CDCl}_{3}\right) \delta 7.33-7.36(\mathrm{~m}, 5 \mathrm{H}), 5.79-5.95$ $(\mathrm{m}, 2 \mathrm{H}), 5.22-5.34(\mathrm{~m}, 2 \mathrm{H}), 5.13(\mathrm{~s}, 2 \mathrm{H}), 4.68-4.75(\mathrm{~m}$, $3 \mathrm{H}), 4.57-4.60(\mathrm{~m}, 2 \mathrm{H}), 3.09\left(\mathrm{dd}, J_{1}=17.1 \mathrm{~Hz}, J_{2}=4.8 \mathrm{~Hz}\right.$, $1 \mathrm{H}), 2.90\left(\mathrm{dd}, J_{1}=17.1 \mathrm{~Hz}, J_{2}=4.5 \mathrm{~Hz}, 1 \mathrm{H}\right), 2.50(\mathrm{t}, J=$ $2.4 \mathrm{~Hz}, 1 \mathrm{H}) ;{ }^{13} \mathrm{C} \mathrm{NMR}\left(\mathrm{CDCl}_{3}\right) \delta 170.3,169.9,155.8,135.9$, $131.5,128.5,128.2,128.0,118.8,76.8,75.5,67.2,65.8,53.2$, 50.3, 36.5; ESMS Calculated for $\mathrm{C}_{18} \mathrm{H}_{19} \mathrm{NO}_{6}+\mathrm{Na}$ : 368.1110, Observed 368.1106.

Boc-Asp(OAllyl)-OPrp (3k). Pale yellow oil; $[\alpha]_{\mathrm{D}} 2009-26$ (c = 1, EtOH); FTIR (Neat) 3382 (s), 2130 (w), 1737 (s); ${ }^{1} \mathrm{H}$ $\operatorname{NMR}\left(\mathrm{CDCl}_{3}\right) \delta 5.82-5.97(\mathrm{~m}, 1 \mathrm{H}), 5.52(\mathrm{~d}, J=8.1 \mathrm{~Hz}$, $1 \mathrm{H}), 5.24-5.36(\mathrm{~m}, 2 \mathrm{H}), 4.74-4.75(\mathrm{~m}, 3 \mathrm{H}), 4.59-4.62(\mathrm{~m}$, $3 \mathrm{H}), 3.06\left(\mathrm{dd}, J_{1}=17.4 \mathrm{~Hz}, J_{2}=4.8 \mathrm{~Hz}, 1 \mathrm{H}\right), 2.88\left(\mathrm{dd}, J_{1}=\right.$ $\left.17.1 \mathrm{~Hz}, J_{2}=4.5 \mathrm{~Hz}, 1 \mathrm{H}\right), 2.50(\mathrm{t}, J=2.7 \mathrm{~Hz}, 1 \mathrm{H}), 1.45(\mathrm{~s}$, $9 \mathrm{H}) ;{ }^{13} \mathrm{C} \mathrm{NMR}\left(\mathrm{CDCl}_{3}\right) \delta 170.4,170.3,155.3,131.6,118.7$, 80.2, 76.6, 75.4, 65.7, 53.1, 49.9, 36.6, 28.2; ESMS Calculated for $\mathrm{C}_{15} \mathrm{H}_{21} \mathrm{NO}_{6}+\mathrm{Na}$ : 334.1267, Observed 334.1249.

Boc-Asp(OPrp)-OMe (3l). Pale yellow oil; $[\alpha]_{\mathrm{D}}-5$ (c = 1, EtOH); FTIR (Neat) 3383 (br), 2131 (w), 1746 (s), 1717 (s); ${ }^{1} \mathrm{H} \mathrm{NMR}\left(\mathrm{CDCl}_{3}\right) \delta 5.55(\mathrm{bd}, J=2.1 \mathrm{~Hz}, 1 \mathrm{H}), 4.68-4.71(\mathrm{~m}$, $2 \mathrm{H}), 4.57-4.65(\mathrm{~m}, 1 \mathrm{H}), 3.76(\mathrm{~s}, 3 \mathrm{H}), 2.85-3.07(\mathrm{~m}, 2 \mathrm{H})$, 2.52-2.55 (m, 1H), $1.45(\mathrm{~s}, 9 \mathrm{H}) ;{ }^{13} \mathrm{C} \mathrm{NMR}\left(\mathrm{CDCl}_{3}\right) \delta 171.2$, 170.0, 155.1, 80.0, 75.2, 52.6, 52.2, 51.8, 49.7, 36.5, 28.1; ESMS Calculated for $\mathrm{C}_{13} \mathrm{H}_{19} \mathrm{NO}_{6}+\mathrm{Na}$ : 308.1110, Observed 308.1110 .

Fmoc-Ala-OPrp (3m). White solid; $m p 88^{\circ} \mathrm{C} ;[\alpha]_{\mathrm{D}}-25$ (c = 1, EtOH); FTIR (Neat) 3401 (br), 2130 (w), 1747 (s), 1724 $(\mathrm{s}) ;{ }^{1} \mathrm{H} \mathrm{NMR}\left(\mathrm{CDCl}_{3}\right) \delta 7.66-7.69(\mathrm{~m}, 2 \mathrm{H}), 7.52-7.56(\mathrm{~m}$, $2 \mathrm{H}), 7.21-7.34(\mathrm{~m}, 4 \mathrm{H}), 5.73(\mathrm{bd}, J=7.5 \mathrm{~Hz}, 1 \mathrm{H}), 4.58-$ $4.70(\mathrm{~m}, 2 \mathrm{H}), 4.31-4.42(\mathrm{~m}, 3 \mathrm{H}), 4.13-4.17(\mathrm{~m}, 1 \mathrm{H}), 2.44$ $(\mathrm{t}, J=2.4 \mathrm{~Hz}, 1 \mathrm{H}), 1.37(\mathrm{~d}, J=6.6 \mathrm{~Hz}, 3 \mathrm{H}) ;{ }^{13} \mathrm{C} \mathrm{NMR}$ $\left(\mathrm{CDCl}_{3}\right) \delta 172.1,155.5,143.4,140.9,127.4,126.7,124.8$, 119.7, 76.8, 75.4, 66.6, 52.5, 49.2, 46.7, 17.8; ESMS Calculated for $\mathrm{C}_{21} \mathrm{H}_{19} \mathrm{NO}_{4}+\mathrm{Na}$ : 372.3697, Observed 372.3701.

Cbz-Asn-OPrp (3n). White solid; mp $123^{\circ} \mathrm{C} ;[\alpha]_{\mathrm{D}}-21$ (c = 1, EtOH); FTIR (Neat) 3292 (br), 2130 (w), 1715 (s); ${ }^{1} \mathrm{H}$ $\operatorname{NMR}\left(\mathrm{CDCl}_{3}\right) \delta 7.30-7.42(\mathrm{~m}, 5 \mathrm{H}), 5.81(\mathrm{bd}, J=6.6 \mathrm{~Hz}$, $1 \mathrm{H}), 5.14(\mathrm{~s}, 2 \mathrm{H}), 4.80(\mathrm{~d}, J=2.1 \mathrm{~Hz}, 2 \mathrm{H}), 4.59-4.65(\mathrm{~m}, 1 \mathrm{H})$, $3.05\left(\mathrm{dd}, J_{1}=17 \mathrm{~Hz}, J_{2}=5 \mathrm{~Hz}, 1 \mathrm{H}\right), 2.95\left(\mathrm{dd}, J_{1}=17 \mathrm{~Hz}\right.$, $\left.J_{2}=5 \mathrm{~Hz}, 1 \mathrm{H}\right), 2.55(\mathrm{t}, J=2.4 \mathrm{~Hz}, 1 \mathrm{H}) ;{ }^{13} \mathrm{C} \mathrm{NMR}\left(\mathrm{CDCl}_{3}\right)$ $\delta$ 168.0, 155.4, 135.6, 128.6, 128.4, 128.1, 115.7, 76.3, 76.1, 67.6, 53.9, 50.5, 21.6; ESMS Calculated for $\mathrm{C}_{15} \mathrm{H}_{16} \mathrm{~N}_{2} \mathrm{O}_{5}+$ Na: 327.2877, Observed 327.2880.

Boc-Tyr (Cbz)-OPrp (3o). White solid; $\mathrm{mp} 60^{\circ} \mathrm{C} ;[\alpha]_{\mathrm{D}}-8$ (c = 1, EtOH); FTIR (Neat) 3292 (br), 2130 (w), 1760 (s), 1715 $(\mathrm{s}) ;{ }^{1} \mathrm{H} \mathrm{NMR}\left(\mathrm{CDCl}_{3}\right) \delta 7.35-7.46(\mathrm{~m}, 5 \mathrm{H}), 7.10-7.19(\mathrm{~m}$,
$4 \mathrm{H}), 5.26(\mathrm{~s}, 2 \mathrm{H}), 4.98(\mathrm{bd}, J=7.5 \mathrm{~Hz}, 1 \mathrm{H}), 4.59-4.80(\mathrm{~m}$, $3 \mathrm{H}), 3.04-3.18(\mathrm{~m}, 2 \mathrm{H}), 2.51(\mathrm{t}, J=2.4 \mathrm{~Hz}, 1 \mathrm{H}), 1.42(\mathrm{~s}$, $9 \mathrm{H}) ;{ }^{13} \mathrm{C} \mathrm{NMR}\left(\mathrm{CDCl}_{3}\right) \delta 170.9,155.0,153.5,150.3,134.8$, $133.6,130.4,128.7,128.5,121.1,80.1,75.5,70.3,54.2,52.6$, 37.4, 28.2; ESMS Calculated for $\mathrm{C}_{25} \mathrm{H}_{27} \mathrm{NO}_{7}+\mathrm{Na}$ : 476.1686, Observed 476.1684.

$\operatorname{Boc}-\operatorname{Ty}(\operatorname{Poc})-\operatorname{OPrp}(3 p)$. White solid; $\mathrm{mp} 65^{\circ} \mathrm{C} ;[\alpha]_{\mathrm{D}}-9$ (c = 1, EtOH); FTIR (Neat) 3290 (br), 2130 (w), 1756 (s), 1710 (s); ${ }^{1} \mathrm{H} \mathrm{NMR}\left(\mathrm{CDCl}_{3}\right) \delta 7.19(\mathrm{~d}, J=8.7 \mathrm{~Hz}, 2 \mathrm{H}), 7.13(\mathrm{~d}, J=$ $8.7 \mathrm{~Hz}, 2 \mathrm{H}), 4.98$ (bd, $J=8 \mathrm{~Hz}, 1 \mathrm{H}), 4.84(\mathrm{~d}, J=2.4 \mathrm{~Hz}, 2 \mathrm{H})$, 4.64-4.80 (m, 3H), 3.09-3.14 (m, 2H), $2.59(\mathrm{t}, J=2.4 \mathrm{~Hz}$, $1 \mathrm{H}), 2.52(\mathrm{t}, J=2.4 \mathrm{~Hz}, 1 \mathrm{H}), 1.42(\mathrm{~s}, 9 \mathrm{H}) ;{ }^{13} \mathrm{C} \mathrm{NMR}\left(\mathrm{CDCl}_{3}\right)$ $\delta 171.3,155.3,153.3,150.5,134.2,130.8,121.3,80.5,76.6$, 76.3, 75.9, 56.2, 54.6, 53.0, 37.8, 28.6; ESMS Calculated for $\mathrm{C}_{21} \mathrm{H}_{23} \mathrm{NO}_{7}+\mathrm{Na}$ : 424.3996, Observed 424.3400.

4.4. General Procedure for the Deprotection of Propargyl Esters Using Benzyltriethylammonium Tetrathiomolybdate ( 1). To a solution of the propargyl esters (3a-o, $1 \mathrm{mmol}$ ) in acetonitrile $(5 \mathrm{~mL})$, benzyltriethylammonium tetrathiomolybdate $(1,1 \cdot 1 \mathrm{mmol}, 0 \cdot 67 \mathrm{~g})$ was added at $\mathrm{rt}\left(28^{\circ} \mathrm{C}\right)$ and the reaction mixture was stirred for $2 \mathrm{~h}$. Acetonitrile was removed under vacuum, and the residue was extracted with a mixture of ethyl acetate and chloroform (9:1). The crude products were purified by column chromatography (silica gel, 100-200 mesh) eluting with a solution of ethyl acetate in petroleum ether or methanol in chloroform. The simultaneous deprotection of the propargyloxycarbonyl group and the propargyl ester in $3 p$ was carried out the same way using 2.1 equiv of 1 .

4.5. General Procedure for the Synthesis of Propargyl Esters (4aj) of Amino Acids. Dry $\mathrm{HCl}$ was bubbled through propargyl alcohol $(20 \mathrm{~mL})$ at $0^{\circ} \mathrm{C}$ for $1 \mathrm{~h}$. The amino acid $(5 \mathrm{mmol})$ is added to the saturated solution of $\mathrm{HCl}$ in propargyl alcohol at $0^{\circ} \mathrm{C}$, and the stirring was continued for $12 \mathrm{~h}$ at $\mathrm{rt}\left(28^{\circ} \mathrm{C}\right)$. Propargyl alcohol is removed under vacuum and the residue is washed with anhydrous diethyl ether $(25 \mathrm{~mL} \times 10)$, dried under vacuum, and stored in a desiccator.

HCl.H-Ala-OPrp (4a). White solid; mp $88^{\circ} \mathrm{C} ;[\alpha]_{\mathrm{D}}+32(\mathrm{c}$ = 1, EtOH); FTIR (KBr) 3292 (br), 2125 (w), 1741 (s); ${ }^{1} \mathrm{H}$ $\operatorname{NMR}\left(\mathrm{D}_{2} \mathrm{O}\right) \delta 4.74(\mathrm{~d}, J=2.4 \mathrm{~Hz}, 2 \mathrm{H}), 4.11(\mathrm{q}, J=7.5 \mathrm{~Hz}$, $1 \mathrm{H}), 2.85(\mathrm{t}, J=2.4 \mathrm{~Hz}, 1 \mathrm{H}), 1.44(\mathrm{~d}, J=7.5 \mathrm{~Hz}, 3 \mathrm{H}) ;{ }^{13} \mathrm{C}$ NMR $\left(\mathrm{D}_{2} \mathrm{O}\right) \delta 170.8,77.7,54.9,49.6,15.8$; ESMS Calculated for $\mathrm{C}_{6} \mathrm{H}_{9} \mathrm{NO}_{2}+\mathrm{H}$ : 128.0633, Observed 128.0701.

$\mathrm{HCl} \cdot \mathrm{H}$-Gly-OPrp (4b). White solid; mp $158^{\circ} \mathrm{C}$; FTIR (KBr) 3440 (br), 2129 (w), 1763 (s); ${ }^{1} \mathrm{H} \operatorname{NMR}\left(\mathrm{D}_{2} \mathrm{O}\right) \delta 4.74$ (d, $J=$ $2.4 \mathrm{~Hz}, 2 \mathrm{H}), 3.84(\mathrm{~s}, 2 \mathrm{H}), 2.83(\mathrm{t}, J=2.4 \mathrm{~Hz}, 1 \mathrm{H}) ;{ }^{13} \mathrm{C} \mathrm{NMR}$ $\left(\mathrm{D}_{2} \mathrm{O}\right) \delta 168.3,101.4,77.6,54.7,40.9$; ESMS Calculated for $\mathrm{C}_{5} \mathrm{H}_{7} \mathrm{NO}_{2}+\mathrm{H}:$ 114.0555, Observed 114.0564.

$\mathrm{HCl} \cdot \mathrm{H}$-Leu-OPrp (4c). White solid; mp $125^{\circ} \mathrm{C} ;[\alpha]_{\mathrm{D}}-19$ (c = 1, EtOH); FTIR (KBr) 3442 (br), 1952 (w), 1749 (s); ${ }^{1} \mathrm{H}$ $\operatorname{NMR}\left(\mathrm{D}_{2} \mathrm{O}\right) \delta 4.76\left(\mathrm{dd}, J_{1}=2.5 \mathrm{~Hz}, J_{2}=1.2 \mathrm{~Hz}, 2 \mathrm{H}\right), 4.07(\mathrm{t}$, $J=7.2 \mathrm{~Hz}, 1 \mathrm{H}), 2.87(\mathrm{t}, J=2.5 \mathrm{~Hz}, 1 \mathrm{H}), 1.59-1.79(\mathrm{~m}, 3 \mathrm{H})$, $0.85(\mathrm{~d}, J=6.3 \mathrm{~Hz}, 3 \mathrm{H}), 0.84(\mathrm{~d}, J=6.3 \mathrm{~Hz}, 3 \mathrm{H}) ;{ }^{13} \mathrm{C} \mathrm{NMR}$ 
$\left(\mathrm{D}_{2} \mathrm{O}\right) \delta 170.9,77.8,77.6,54.9,52.2,39.5,24.8,22.3,21.9$; ESMS Calculated for $\mathrm{C}_{9} \mathrm{H}_{15} \mathrm{NO}_{2}+\mathrm{H}: 170.1182$, Observed 170.1179 .

$\mathrm{HCl} \cdot \mathrm{H}$-Pro-OPrp (4d). White solid; $\mathrm{mp} 52^{\circ} \mathrm{C}$; $[\alpha]_{\mathrm{D}}-76$ (c = 1, EtOH); FTIR (KBr) 3410 (br), 2123 (w), 1751 (s); ${ }^{1} \mathrm{H}$ $\operatorname{NMR}\left(\mathrm{D}_{2} \mathrm{O}\right) \delta 4.77(\mathrm{~d}, J=2.1 \mathrm{~Hz}, 2 \mathrm{H}), 4.42\left(\mathrm{dd}, J_{1}=8.6 \mathrm{~Hz}\right.$, $\left.J_{2}=7.2 \mathrm{~Hz}, 1 \mathrm{H}\right), 3.25-3.39(\mathrm{~m}, 2 \mathrm{H}), 2.88(\mathrm{t}, J=2.4 \mathrm{~Hz}, 1 \mathrm{H})$, $2.29-2.41(\mathrm{~m}, 1 \mathrm{H}), 1.90-2.14(\mathrm{~m}, 3 \mathrm{H}) ;{ }^{13} \mathrm{C} \mathrm{NMR}\left(\mathrm{D}_{2} \mathrm{O}\right) \delta$ 170.0, 77.8, 77.6, 60.2, 55.2, 47.2, 28.9, 24.1; ESMS Calculated for $\mathrm{C}_{8} \mathrm{H}_{11} \mathrm{NO}_{2}+\mathrm{H}$ : 154.0790, Observed 154.0860.

$\mathrm{HCl} \cdot \mathrm{H}$-Ser-OPrp (4e). Pale yellow oil; $[\alpha]_{\mathrm{D}}-40 \quad(\mathrm{c}=1$, EtOH); FTIR (Neat) 3434 (br), 2129 (w), 1751 (s); ${ }^{1} \mathrm{H}$ NMR $\left(\mathrm{D}_{2} \mathrm{O}\right) \delta 4.76-4.77(\mathrm{~m}, 2 \mathrm{H}), 4.18-4.21(\mathrm{~m}, 1 \mathrm{H}), 4.00$ (ddd, $J_{1}$ $\left.=13 \mathrm{~Hz}, J_{2}=4.5 \mathrm{~Hz}, J_{3}=1.8 \mathrm{~Hz}, 1 \mathrm{H}\right), 3.83-3.92(\mathrm{~m}, 1 \mathrm{H})$, $2.85\left(\mathrm{dd}, J_{1}=4.6 \mathrm{~Hz}, J_{2}=2.7 \mathrm{~Hz}, 1 \mathrm{H}\right) ;{ }^{13} \mathrm{C} \operatorname{NMR}\left(\mathrm{D}_{2} \mathrm{O}\right)$ $\delta 168.6,77.7,77.5,59.9,55.5,55.1$; ESMS Calculated for $\mathrm{C}_{6} \mathrm{H}_{9} \mathrm{NO}_{3}+\mathrm{Na}:$ 166.0480, Observed 166.0491.

$\mathrm{HCl} \cdot \mathrm{H}$-Phe-OPrp (4f). White solid; mp $154^{\circ} \mathrm{C} ;[\alpha]_{\mathrm{D}}-40$ (c = 1, EtOH); FTIR (KBr) 3288 (br), 2127 (w), 1743 (s); ${ }^{1} \mathrm{H}$ $\operatorname{NMR}\left(\mathrm{CDCl}_{3}\right) \delta 7.11-7.24(\mathrm{~m}, 5 \mathrm{H}), 4.63(\mathrm{~d}, J=2.1 \mathrm{~Hz}, 2 \mathrm{H})$, $3.68\left(\mathrm{dd}, J_{1}=7.8 \mathrm{~Hz}, J_{2}=5.1 \mathrm{~Hz}, 1 \mathrm{H}\right), 3.02\left(\mathrm{dd}, J_{1}=13.5 \mathrm{~Hz}\right.$, $\left.J_{2}=5.1 \mathrm{~Hz}, 1 \mathrm{H}\right), 2.81\left(\mathrm{dd}, J_{1}=13.5 \mathrm{~Hz}, J_{2}=7.8 \mathrm{~Hz}, 1 \mathrm{H}\right), 2.43$ $\left(\mathrm{dd}, J_{1}=2.7 \mathrm{~Hz}, J_{2}=1.8 \mathrm{~Hz}, 1 \mathrm{H}\right), 1.49$ (bs, $\left.2 \mathrm{H}\right) ;{ }^{13} \mathrm{C} \mathrm{NMR}$ $\left(\mathrm{CDCl}_{3}\right) \delta 174.1,136.7,129.2,128.4,126.7,77.2,75.1,55.5$, 52.2, 40.6; ESMS Calculated for $\mathrm{C}_{12} \mathrm{H}_{13} \mathrm{NO}_{2}+\mathrm{H}$ : 204.1030, Observed 204.1024.

$\mathrm{HCl} \cdot \mathrm{H}-\mathrm{Glu}(\mathrm{OPrp})-\mathrm{OPrp}$ (4g). White solid; mp $119^{\circ} \mathrm{C}$; $[\alpha]_{\mathrm{D}}+50(\mathrm{c}=1, \mathrm{EtOH})$; FTIR $(\mathrm{KBr}) 3435(\mathrm{br}), 2132(\mathrm{w})$, $1740(\mathrm{~s}) ;{ }^{1} \mathrm{H}$ NMR $\left(\mathrm{D}_{2} \mathrm{O}\right) \delta 4.71-4.72(\mathrm{~m}, 2 \mathrm{H}), 4.58-4.60(\mathrm{~m}$, $2 \mathrm{H}), 4.04-4.12(\mathrm{~m}, 1 \mathrm{H}), 2.83\left(\mathrm{dd}, J_{1}=5.1 \mathrm{~Hz}, J_{2}=2.7 \mathrm{~Hz}\right.$, $1 \mathrm{H}), 2.76\left(\mathrm{dd}, J_{1}=5.1 \mathrm{~Hz}, J_{2}=2.7 \mathrm{~Hz}, 1 \mathrm{H}\right), 2.50-2.56(\mathrm{~m}$, 2H), 2.03-2.22 (m, 2H); ${ }^{13} \mathrm{C} \operatorname{NMR}\left(\mathrm{D}_{2} \mathrm{O}\right) \delta$ 174.1, 169.7, 77.8, 77.4, 76.9, 55.1, 53.7, 52.6, 30.0, 25.4; ESMS Calculated for $\mathrm{C}_{11} \mathrm{H}_{13} \mathrm{NO}_{4}+\mathrm{Na}$ : 246.0742, Observed 246.0730.

$\mathrm{HCl} \cdot \mathrm{H}$-Thr-OPrp (4h). Pale yellow oil; $[\alpha]_{\mathrm{D}}-26(\mathrm{c}=1$, EtOH); FTIR (Neat) 3381 (br), 2128 (w), 1751 (s); ${ }^{1} \mathrm{H}$ NMR $\left(\mathrm{D}_{2} \mathrm{O}\right) \delta 4.76(\mathrm{~d}, J=1.8 \mathrm{~Hz}, 2 \mathrm{H}), 4.26-4.33(\mathrm{~m}, 1 \mathrm{H}), 4.01$ $(\mathrm{d}, J=3.6 \mathrm{~Hz}, 1 \mathrm{H}), 2.84(\mathrm{t}, J=1.8 \mathrm{~Hz}, 1 \mathrm{H}), 1.20(\mathrm{~d}, J=$ $6.6 \mathrm{~Hz}, 3 \mathrm{H}) ;{ }^{13} \mathrm{C} \mathrm{NMR}\left(\mathrm{D}_{2} \mathrm{O}\right) \delta 168.8,77.8,77.5,66.0,59.2$, 55.1, 19.7; ESMS Calculated for $\mathrm{C}_{7} \mathrm{H}_{11} \mathrm{NO}_{3}+\mathrm{Na}$ : 180.0637, Observed 180.0640 .

H-4Aba-OPrp (4i). White solid; mp $85^{\circ} \mathrm{C}$; FTIR (KBr) 3432 (br), $2125(\mathrm{w}), 1694(\mathrm{~s}) ;{ }^{1} \mathrm{H}$ NMR $\left(\mathrm{CDCl}_{3}\right) \delta 7.88(\mathrm{~d}, J=$ $8.7 \mathrm{~Hz}, 2 \mathrm{H}), 6.40(\mathrm{~d}, J=2.4 \mathrm{~Hz}, 2 \mathrm{H}), 4.87(\mathrm{~d}, J=2.4 \mathrm{~Hz}$, $2 \mathrm{H}), 4.11$ (bs, 2H), $2.49(\mathrm{t}, J=2.4 \mathrm{~Hz}, 1 \mathrm{H}) ;{ }^{13} \mathrm{C} \mathrm{NMR}$ $\left(\mathrm{CDCl}_{3}\right) \delta 151.2,148.0,131.9,118.7,113.8,78.2$, 74.5, 51.9.; ESMS Calculated for $\mathrm{C}_{10} \mathrm{H}_{9} \mathrm{NO}_{2}+\mathrm{Na}$ : 198.0531, Observed 198.0525 .

H-3Aba-OPrp (4j). Pale yellow oil; FTIR (Neat) 3368 (br), $2127(\mathrm{w}), 1716(\mathrm{~s}) ;{ }^{1} \mathrm{H} \mathrm{NMR}\left(\mathrm{CDCl}_{3}+\mathrm{DMSO}-d_{6}\right) \delta 7.35-$ $7.41(\mathrm{~m}, 2 \mathrm{H}), 7.20(\mathrm{t}, J=7.8 \mathrm{~Hz}, 1 \mathrm{H}), 6.87-6.90(\mathrm{~m}, 1 \mathrm{H})$, $4.88(\mathrm{~d}, J=2.7 \mathrm{~Hz}, 2 \mathrm{H}), 4.12$ (bs, $2 \mathrm{H}), 2.57(\mathrm{t}, J=2.7 \mathrm{~Hz}$,
$1 \mathrm{H}) ;{ }^{13} \mathrm{C} \mathrm{NMR}\left(\mathrm{CDCl}_{3}+\mathrm{DMSO}-d_{6}\right) \delta 165.6,146.7,129.8$, 128.9, 119.4, 118.9, 115.3, 77.4, 74.8, 51.9; ESMS Calculated for $\mathrm{C}_{10} \mathrm{H}_{9} \mathrm{NO}_{2}+\mathrm{H}$ : 176.0711, Observed 176.0709.

4.6. Synthesis of Peptides $5 \boldsymbol{a}-\boldsymbol{c}$. Boc-amino propargyl esters ( $2 \mathbf{c}$, $\mathbf{d}$ and $\mathbf{g}, 2 \mathrm{mmol}$ ) were dissolved in $5 \mathrm{~mL}$ solution of TFA (50\%) in $\mathrm{CH}_{2} \mathrm{Cl}_{2}$. The solution was stirred at $\mathrm{rt}\left(28^{\circ} \mathrm{C}\right)$ for $1 \mathrm{~h}$ and then concentrated under vacuum. The crude TFA salt of the amino propargyl esters were then dissolved in acetonitrile and used for peptide coupling without further purification.

The trifluoroacetate salt of the amino propargyl ester ( $2 \mathrm{mmol}$, obtained as above), an $N$-protected amino acid $(2 \mathrm{mmol})$ and $\mathrm{HOBt}(0.270 \mathrm{~g}, 2 \mathrm{mmol})$ were dissolved in acetonitrile $(15 \mathrm{~mL})$. The solution was cooled to $0^{\circ} \mathrm{C}$ and $N$-methylmorpholine $(0.24 \mathrm{~mL}, 2.2 \mathrm{mmol})$ was added dropwise. A solution of DCC $(0.62 \mathrm{~g}, 3 \mathrm{mmol})$ in acetonitrile $(5 \mathrm{~mL})$ was added to the reaction mixture. Reaction mixture was allowed to come to $\mathrm{rt}\left(28^{\circ} \mathrm{C}\right)$, and stirring was continued for $4 \mathrm{~h}$. The solvent was removed under vacuum, and the residue was extracted with cold ethyl acetate $(50 \mathrm{~mL})$ and filtered over a celite pad. The ethyl acetate solution was washed with saturated citric acid solution $(40 \mathrm{~mL})$, saturated $\mathrm{Na}_{2} \mathrm{CO}_{3}$ solution $(40 \mathrm{~mL})$, and finally with brine $(40 \mathrm{~mL})$. The crude solution of the peptide was dried over anhydrous $\mathrm{Na}_{2} \mathrm{SO}_{4}$ and concentrated. The peptides $(\mathbf{5 a}-\mathbf{c})$ were purified by column chromatography (silica gel, 100-200 mesh) eluting with a solution of ethyl acetate $(20-40 \%)$ in petroleum ether.

Boc-Ile-Aib-OPrp (5a). White solid; mp $117^{\circ} \mathrm{C} ;[\alpha]_{\mathrm{D}}-31$ (c = 1, EtOH); FTIR (Neat) 3311 (br), 2133 (w), 1748 (s), 1684 (s), $1652(\mathrm{~s}) ;{ }^{1} \mathrm{H}$ NMR $\left(\mathrm{CDCl}_{3}\right) \delta 6.57$ (bs, $\left.1 \mathrm{H}\right), 5.08$ (bd, $J=$ $8 \mathrm{~Hz}, 1 \mathrm{H}), 4.71(\mathrm{~d}, J=2.7 \mathrm{~Hz}, 2 \mathrm{H}), 3.87-3.92(\mathrm{~m}, 1 \mathrm{H}), 2.46$ $(\mathrm{t}, J=2.7 \mathrm{~Hz}, 1 \mathrm{H}), 1.85-1.87(\mathrm{~m}, 1 \mathrm{H}), 1.55(\mathrm{~d}, J=2.1 \mathrm{~Hz}$, $6 \mathrm{H}), 1.45(\mathrm{~s}, 9 \mathrm{H}), 1.02-1.21(\mathrm{~m}, 1 \mathrm{H}), 0.89-0.95(\mathrm{~m}, 6 \mathrm{H})$; ${ }^{13} \mathrm{C} \mathrm{NMR}\left(\mathrm{CDCl}_{3}\right) \delta 173.3,170.8,155.9,79.9,75.0,59.2$, 56.2, 52.8, 37.0, 28.3, 24.7, 15.4, 11.3; ESMS Calculated for $\mathrm{C}_{18} \mathrm{H}_{30} \mathrm{~N}_{2} \mathrm{O}_{5}+\mathrm{Na}$ : 377.2053, Observed 377.2050.

Boc-Val-Val-OPrp (5b). White solid; mp $89^{\circ} \mathrm{C} ;[\alpha]_{\mathrm{D}}-55$ (c = 1, EtOH); FTIR (Neat) 3308 (br), 2130 (w), 1749 (s), 1686 (s), $1653(\mathrm{~s}) ;{ }^{1} \mathrm{H} \mathrm{NMR}\left(\mathrm{CDCl}_{3}\right) \delta 6.56(\mathrm{bd}, J=8.4 \mathrm{~Hz}, 1 \mathrm{H})$, $5.18(\mathrm{bd}, J=8.7 \mathrm{~Hz}, 1 \mathrm{H}), 4.79\left(\mathrm{dd}, J_{1}=15.6 \mathrm{~Hz}, J_{2}=2.4 \mathrm{~Hz}\right.$, $1 \mathrm{H}), 4.67\left(\mathrm{dd}, J_{1}=15.6 \mathrm{~Hz}, J_{2}=2.4 \mathrm{~Hz}, 1 \mathrm{H}\right), 4.56-4.60(\mathrm{~m}$, $1 \mathrm{H}), 3.92-3.97(\mathrm{~m}, 1 \mathrm{H}), 2.50(\mathrm{t}, J=2.4 \mathrm{~Hz}, 1 \mathrm{H}), 2.08-2.28$ $(\mathrm{m}, 2 \mathrm{H}), 1.44(\mathrm{~s}, 9 \mathrm{H}), 0.95(\mathrm{t}, J=7.5 \mathrm{~Hz}, 12 \mathrm{H}) ;{ }^{13} \mathrm{C} \mathrm{NMR}$ $\left(\mathrm{CDCl}_{3}\right) \delta 171.8,170.9,155.8,79.7,75.2,60.0,56.9,52.4$, 31.1, 30.5, 28.2, 19.2, 18.8, 17.9, 17.6; ESMS Calculated for $\mathrm{C}_{18} \mathrm{H}_{30} \mathrm{~N}_{2} \mathrm{O}_{5}+\mathrm{Na}$ : 377.2053, Observed 377.2055.

Boc-Phe-Ile-OPrp (5c). White solid; mp $109^{\circ} \mathrm{C} ;[\alpha]_{\mathrm{D}}-21$ (c = 1, EtOH); FTIR (Neat) 3283 (br), 2136 (w), 1744 (s), 1683 (s), 1658 (s); ${ }^{1} \mathrm{H}$ NMR $\left(\mathrm{CDCl}_{3}\right) \delta$ 7.21-7.32 (m, 5H), $6.40(\mathrm{bd}, J=7.2 \mathrm{~Hz}, 1 \mathrm{H}), 5.07$ (bs, $1 \mathrm{H}), 4.72\left(\mathrm{dd}, J_{1}=\right.$ $\left.15.5 \mathrm{~Hz}, J_{2}=2.4 \mathrm{~Hz}, 1 \mathrm{H}\right), 4.65\left(\mathrm{dd}, J_{1}=15.7 \mathrm{~Hz}, J_{2}=2.4 \mathrm{~Hz}\right.$, $1 \mathrm{H}), 4.51-4.56(\mathrm{~m}, 1 \mathrm{H}), 4.32-4.39(\mathrm{~m}, 1 \mathrm{H}), 3.07(\mathrm{~d}, J=$ $6.9 \mathrm{~Hz}, 2 \mathrm{H}), 2.49(\mathrm{t}, J=2.4 \mathrm{~Hz}, 1 \mathrm{H}), 1.76-1.89(\mathrm{~m}, 1 \mathrm{H})$, $1.33-1.49(\mathrm{~m}, 10 \mathrm{H}), 1.04-1.26(\mathrm{~m}, 1 \mathrm{H}), 0.84-0.91(\mathrm{~m}, 6 \mathrm{H})$; 
${ }^{13} \mathrm{C} \mathrm{NMR}\left(\mathrm{CDCl}_{3}\right) \delta 171.0,170.5,155.4,136.6,129.3,128.6$, 126.9, 80.2, 75.3, 56.4, 55.7, 52.3, 37.9, 28.2, 25.0, 15.2, 11.4; ESMS Calculated for $\mathrm{C}_{23} \mathrm{H}_{32} \mathrm{~N}_{2} \mathrm{O}_{5}+\mathrm{Na}: 439.2209$, Observed 439.2212 .

4.7. Synthesis of the Tetrapeptide 9. The dipeptide 6 was prepared using $3 \mathrm{mmol}$ each of the protected amino acids BocAla-OH $(0.57 \mathrm{~g})$ and $\mathrm{HCl} \cdot \mathrm{H}-\mathrm{Phe}-\mathrm{OPrp}(0.72 \mathrm{~g})$. The peptide coupling was carried out as described for the preparation of $\mathbf{5 a}-\mathbf{c}$, and $\mathbf{6}$ was obtained in $92 \%$ yield $(1.03 \mathrm{~g})$. The Boc group was deprotected from $6(0.375 \mathrm{~g}, 1 \mathrm{mmol})$ to get 7 using 50\% TFA, and the propargyl ester was deprotected from $6(0.375 \mathrm{~g}, 1 \mathrm{mmol})$ to get 8 using tetrathiomolybdate (1). The compounds 7 and $\mathbf{8}$ were coupled using DCC to get the tetrapeptide 9 in $80 \%$ yield $(0.47 \mathrm{~g})$ after purification using silica gel column chromatography.

Boc-Ala-Phe-OPrp (6). White solid; mp $81^{\circ} \mathrm{C} ;[\alpha]_{\mathrm{D}}-31$ (c = 1, EtOH); FTIR (Neat) 3297 (br), 2124 (w), 1752 (s), 1708 (s), 1687 (s); ${ }^{1} \mathrm{H} \mathrm{NMR}\left(\mathrm{CDCl}_{3}\right) \delta 7.24-7.32(\mathrm{~m}, 3 \mathrm{H}), 7.13-$ 7.16 (m, 2H), 6.59 (bd, $J=7.5 \mathrm{~Hz}, 1 \mathrm{H}), 4.99$ (bs, 1H), 4.85$4.92(\mathrm{~m}, 1 \mathrm{H}), 4.76\left(\mathrm{dd}, J_{1}=15.5 \mathrm{~Hz}, J_{2}=2.1 \mathrm{~Hz}, 1 \mathrm{H}\right), 4.67$ $\left(\mathrm{dd}, J_{1}=15.6 \mathrm{~Hz}, J_{2}=2.1 \mathrm{~Hz}, 1 \mathrm{H}\right), 4.08-4.17(\mathrm{~m}, 1 \mathrm{H}), 3.19$ $\left(\mathrm{dd}, J_{1}=14 \mathrm{~Hz}, J_{2}=6 \mathrm{~Hz}, 1 \mathrm{H}\right), 3.11\left(\mathrm{dd}, J_{1}=14 \mathrm{~Hz}, J_{2}=6 \mathrm{~Hz}\right.$, $1 \mathrm{H}), 2.52(\mathrm{t}, J=2.4 \mathrm{~Hz}, 1 \mathrm{H}), 1.43(\mathrm{~s}, 9 \mathrm{H}), 1.31(\mathrm{~d}, J=6.9 \mathrm{~Hz}$, $3 \mathrm{H}) ;{ }^{13} \mathrm{C} \mathrm{NMR}\left(\mathrm{CDCl}_{3}\right) \delta 172.3,170.5,155.3,135.4,129.4$, 128.6, 127.2, 80.1, 76.9, 75.5, 53.0, 52.7, 50.1, 37.6, 28.2, 18.2; ESMS Calculated for $\mathrm{C}_{20} \mathrm{H}_{26} \mathrm{~N}_{2} \mathrm{O}_{5}+\mathrm{Na}$ : 397.1740, Observed 397.1737.

Boc-Ala-Phe-Ala-Phe-OPrp (9). White solid; mp $194^{\circ} \mathrm{C}$; $[\alpha]_{\mathrm{D}}-24(\mathrm{c}=1, \mathrm{EtOH}) ;$ FTIR $(\mathrm{KBr}) 3295(\mathrm{br}), 2130(\mathrm{w})$, 1750 (s), 1711 (s), 1656 (s); ${ }^{1} \mathrm{H}$ NMR (DMSO-d D $\delta 8.30$ (bd, $J=7.2 \mathrm{~Hz}, 1 \mathrm{H}), 8.09(\mathrm{bd}, J=7.2 \mathrm{~Hz}, 1 \mathrm{H}), 7.67$ (bd, $J=8 \mathrm{~Hz}, 1 \mathrm{H}), 6.99-7.07(\mathrm{~m}, 10 \mathrm{H}), 6.76(\mathrm{bd}, J=7.2 \mathrm{~Hz}$, $1 \mathrm{H}), 4.48(\mathrm{~s}, 2 \mathrm{H}), 4.27-4.34(\mathrm{~m}, 2 \mathrm{H}), 4.08-4.12(\mathrm{~m}, 1 \mathrm{H})$, $3.64-3.71(\mathrm{~m}, 1 \mathrm{H}), 2.77-2.84(\mathrm{~m}, 3 \mathrm{H}), 2.53-2.60(\mathrm{~m}, 1 \mathrm{H})$, 1.15 (s, 9H), 0.99 (d, $J=6.6 \mathrm{~Hz}, 3 \mathrm{H}), 0.86(\mathrm{~d}, J=7.2 \mathrm{~Hz}$, $3 \mathrm{H}) ;{ }^{13} \mathrm{C}$ NMR (DMSO- $\left.d_{6}\right) \delta 172.6,172.3,170.51,170.47$, $155.0,137.6,136.8,128.3,129.1,128.3,127.9,126.6,126.2$, 78.2, 78.0, 77.8, 53.5, 53.4, 52.3, 50.0, 48.0, 37.5, 36.4, 28.1, 18.2, 18.1; ESMS Calculated for $\mathrm{C}_{32} \mathrm{H}_{40} \mathrm{~N}_{4} \mathrm{O}_{7}+\mathrm{Na}$ : 615.2795, Observed 615.2772.

\section{Acknowledgments}

R. Ramapanicker thanks CSIR and R. Gupta and R. Megha thank DRDO, New Delhi for the research fellowships.

\section{References}

[1] A. Isidro-Llobet, M. Álvarez, and F. Albericio, "Amino acidprotecting groups," Chemical Reviews, vol. 109, no. 6, pp. 2455-2504, 2009.

[2] K. R. Prabhu, N. Devan, and S. Chandrasekaran, "The Chemistry of tetrathiomolybdate: applications in organic synthesis," Synlett, no. 11, pp. 1762-1778, 2002.

[3] V. M. Swamy, P. Ilankumaran, and S. Chandrasekaran, "Selective deprotection of propargyl ethers using tetrathiomolybdate," Synlett, no. 1997, pp. 513-514, 1997.
[4] P. Ilankumaran, N. Manoj, and S. Chandrasekaran, "Prop-2ynyl as a protective group for carboxylic acids: a mild method for the highly selective deprotection of prop-2-ynyl esters using tetrathiomolybdate," Chemical Communications, no. 16, pp. 1957-1958, 1996.

[5] S. Sinha, P. Ilankumaran, and S. Chandrasekaran, "The prop-2-ynyloxy carbonyl function (POC): a new aminoprotecting group removable from sulfur-containing peptides by ultrasonic irradiation with tetrathiomolybdate under mild and neutral conditions," Tetrahedron Letters, vol. 40, no. 4, pp. 771-774, 1999.

[6] R. G. Bhat, S. Sinha, and S. Chandrasekaran, "Propargyloxycarbonyl (Poc) amino acid chlorides as efficient coupling reagents for the synthesis of $100 \%$ diastereopure peptides and resin bound tetrathiomolybdate as an effective deblocking agent for the Poc group," Chemical Communications, no. 8, pp. 812-813, 2002.

[7] P. R. Sridhar and S. Chandrasekaran, "Propargyloxycarbonyl (Poc) as a protective group for the hydroxyl function in carbohydrate synthesis," Organic Letters, vol. 4, no. 26, pp. 4731-4733, 2002.

[8] R. Ramesh, R. G. Bhat, and S. Chandrasekaran, "Highly selective deblocking of propargyl carbonates in the presence of propargyl carbamates with tetrathiomolybdate," Journal of Organic Chemistry, vol. 70, no. 3, pp. 837-840, 2005.

[9] R. Ramesh, K. De, S. Gupta, and S. Chandrasekaran, "Propargyloxycarbonyl as a protecting group for the side chains of serine, threonine and tyrosine," Journal of Chemical Sciences, vol. 120, no. 1, pp. 163-173, 2008.

[10] S. Kim, J. I. Lee, and Y. C. Kim, "A simple and mild esterification method for carboxylic acids using mixed carboxyliccarbonic anhydrides," Journal of Organic Chemistry, vol. 50, no. 5, pp. 560-565, 1985, The conditions used for propargyloxycarbonyl protection using $\mathrm{PocCl}$ yields propargyl esters in the presence of a strong base such as triethylamine. 

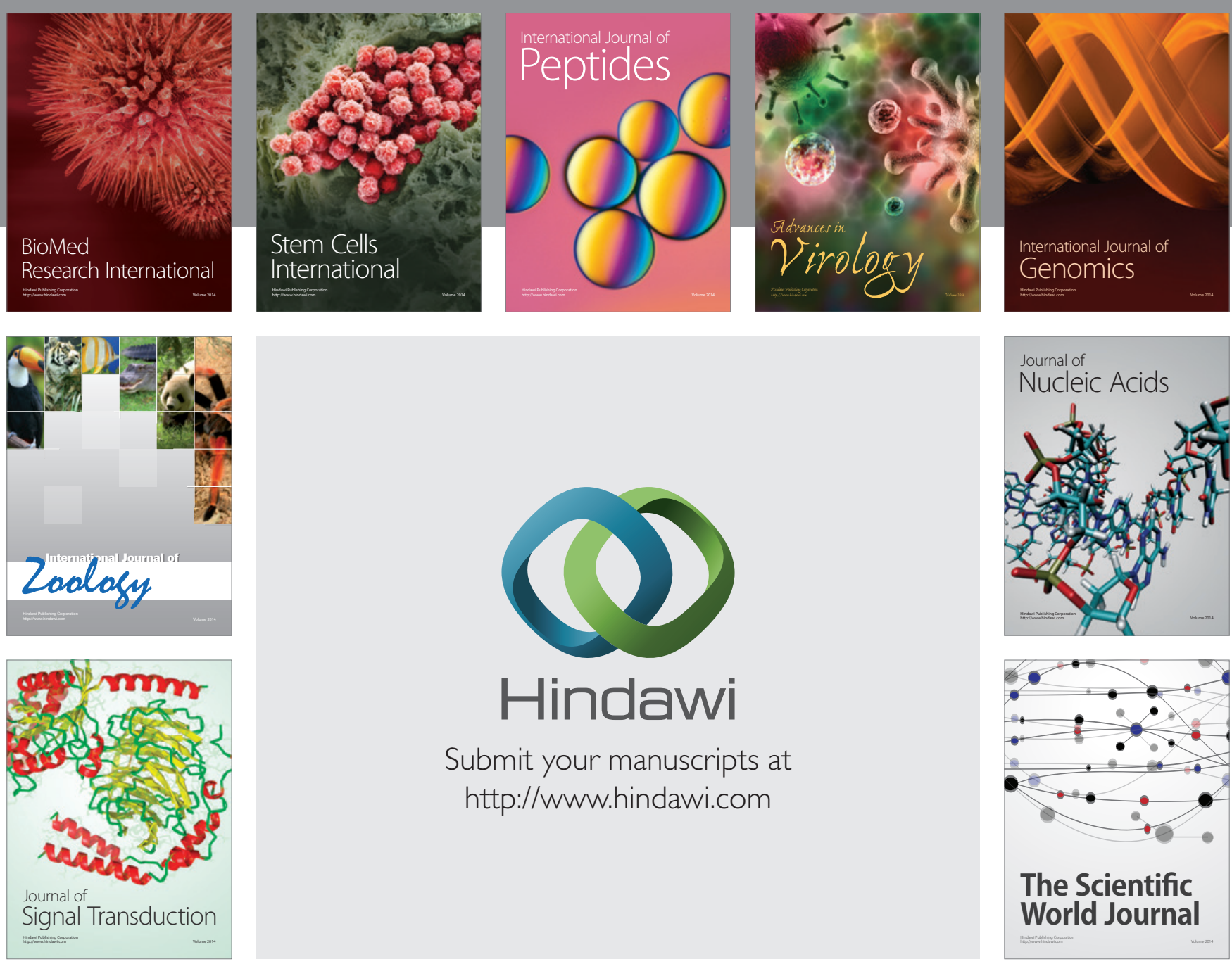

Submit your manuscripts at

http://www.hindawi.com
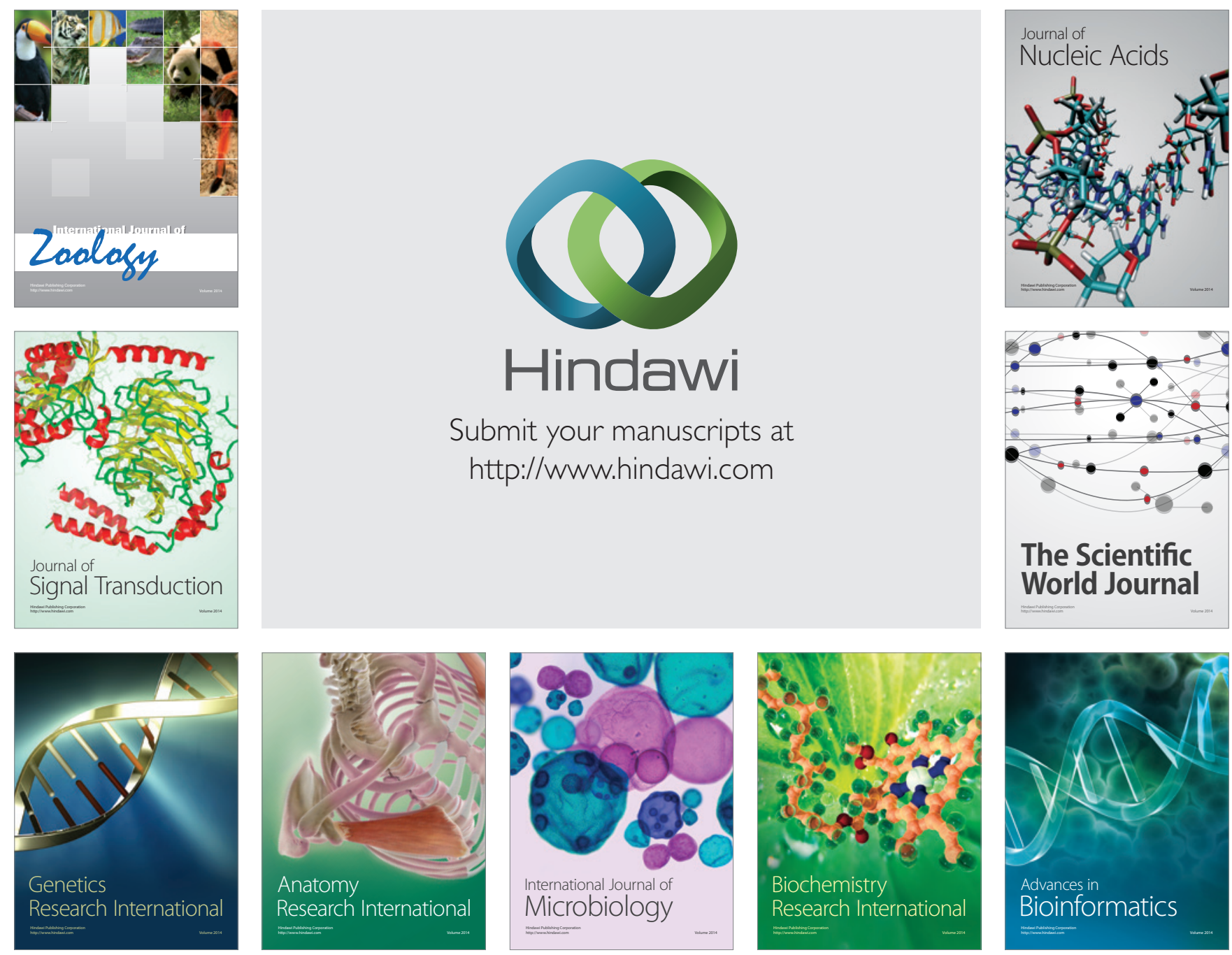

The Scientific World Journal
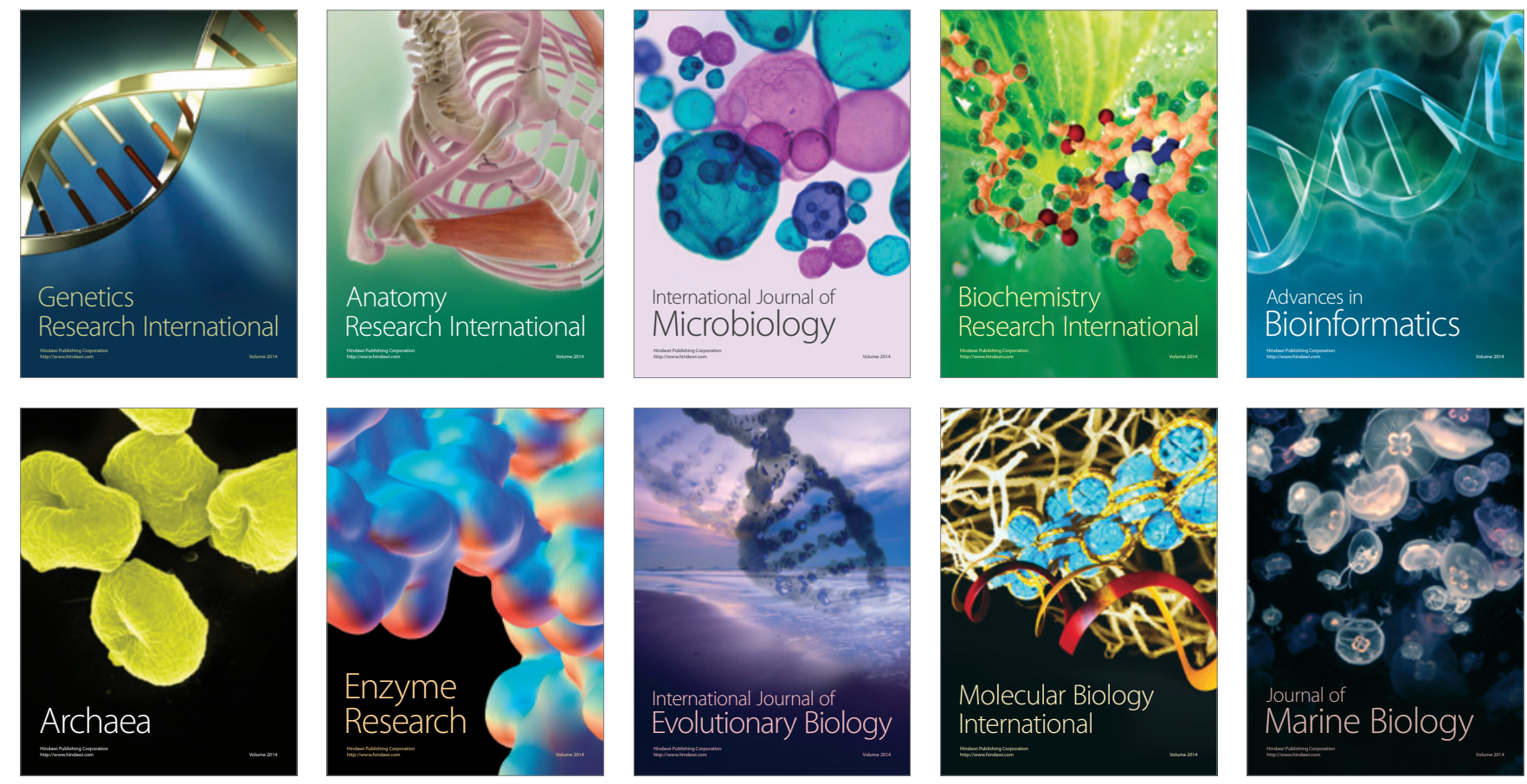